\title{
Temporal-Pattern Recognition by Single Neurons in a Sensory Pathway Devoted to Social Communication Behavior
}

\author{
Bruce A. Carlson \\ Department of Biology, Washington University in St. Louis, St. Louis, Missouri 63130, and Department of Neurobiology and Behavior, Cornell University, \\ Ithaca, New York 14853
}

\begin{abstract}
Sensory systems often encode stimulus information into the temporal pattern of action potential activity. However, little is known about how the information contained within these patterns is extracted by postsynaptic neurons. Similar to temporal coding by sensory neurons, social information in mormyrid fish is encoded into the temporal patterning of an electric organ discharge. In the current study, sensitivity to temporal patterns of electrosensory stimuli was found to arise within the midbrain posterior exterolateral nucleus (ELp). Whole-cell patch recordings from ELp neurons in vivo revealed three patterns of interpulse interval (IPI) tuning: low-pass neurons tuned to long intervals, high-pass neurons tuned to short intervals, and bandpass neurons tuned to intermediate intervals. Many neurons within each class also responded preferentially to either increasing or decreasing IPIs. Playback of electric signaling patterns recorded from freely behaving fish revealed that the IPI and direction tuning of ELp neurons resulted in selective responses to particular social communication displays characterized by distinct IPI patterns. The postsynaptic potential responses of many neurons indicated a combination of excitatory and inhibitory synaptic input, and the IPI tuning of ELp neurons was directly related to rate-dependent changes in the direction and amplitude of postsynaptic potentials. These results suggest that differences in the dynamics of short-term synaptic plasticity in excitatory and inhibitory pathways may tune central sensory neurons to particular temporal patterns of presynaptic activity. This may represent a general mechanism for the processing of behaviorally relevant stimulus information encoded into temporal patterns of activity by sensory neurons.
\end{abstract}

\section{Introduction}

Temporal structure is an essential feature of sensory input. As a result, stimulus-dependent temporal structure in neuronal responses is a ubiquitous feature of sensory systems, which are often characterized by anatomical and physiological specializations devoted to precise temporal coding (Carr, 1993; Carr and Soares, 2002). In addition to conveying information about the precise timing of sensory events, timing can be used by the nervous system as a code in its purest sense, meaning that nontemporal information about stimuli can be represented by precise temporal patterns of activity (Theunissen and Miller, 1995; Rieke et al., 1997; Grothe and Klump, 2000; Lestienne, 2001).

A central problem for temporal coding is determining how the information contained within temporal patterns of activity can be decoded, or extracted, by postsynaptic neurons (Eggermont, 1998; Grothe and Klump, 2000; Lestienne, 2001; Abbott and Regehr, 2004). Primarily because of research in sound localization and electrosensory pathways, we have a basic understanding of neuronal

Received April 27, 2009; revised June 8, 2009; accepted June 24, 2009.

This work was supported by National Institute on Deafness and Other Communication Disorders Grant R01 DC006206-23 (C. D. Hopkins), National Science Foundation Grant I0S0818390 (B.A.C.), and the McDonnell Center for Systems Neuroscience (B.A.C.). Thanks to M. Kawasaki and C. D. Hopkins for support and feedback.

Correspondence should be addressed to Bruce A. Carlson, Washington University in St. Louis, Department of Biology, 1 Brookings Drive, Campus Box 1137, St. Louis, M0 63130-4899. E-mail: carlson.bruce@wustl.edu. DOI:10.1523/JNEUROSCI.1980-09.2009

Copyright $\odot 2009$ Society for Neuroscience $\quad$ 0270-6474/09/299417-12\$15.00/0 mechanisms for detecting submillisecond differences in spike timing between neurons, although many questions remain (Jeffress, 1948; Carr, 1986; Xu-Friedman and Hopkins, 1999; Grothe, 2003; Carlson, 2008). However, we know much less about how information encoded into distinct patterns of activity over time-coding schemes based on sequences of interspike intervals within neurons rather than differences in spike timing between neurons-is decoded by postsynaptic neurons. Theoretical treatments have suggested a number of possibilities (Buonomano, 2000; Cariani, 2001; Large and Crawford, 2002), and in vitro brain slice studies have yielded significant insight into synaptic physiology (Abbott and Regehr, 2004). Although these in vitro studies have revealed many different ways in which synapses can filter presynaptic spike trains, the function of this filtering in the processing of behaviorally relevant, temporally coded sensory information remains unclear.

Communication behavior in mormyrid electric fish from Africa (Fig. $1 A$ ) is itself a temporal code analogous to temporal coding in the nervous system: an all-or-none electrical potential [the electric organ discharge (EOD)] produced with variable timing [the sequence of pulse intervals (SPI)] (Fig. $1 B$ ). By varying the SPI during signaling, mormyrids are able to produce a rich repertoire of electric displays that play important roles in social behavior (Hopkins, 1986; Carlson, 2002; Carlson and Hopkins, 2004; Arnegard and Carlson, 2005; Wong and Hopkins, 2007).

Electric communication is mediated exclusively by Knollenorgan receptors, which are specialized "communication sensors" 
A
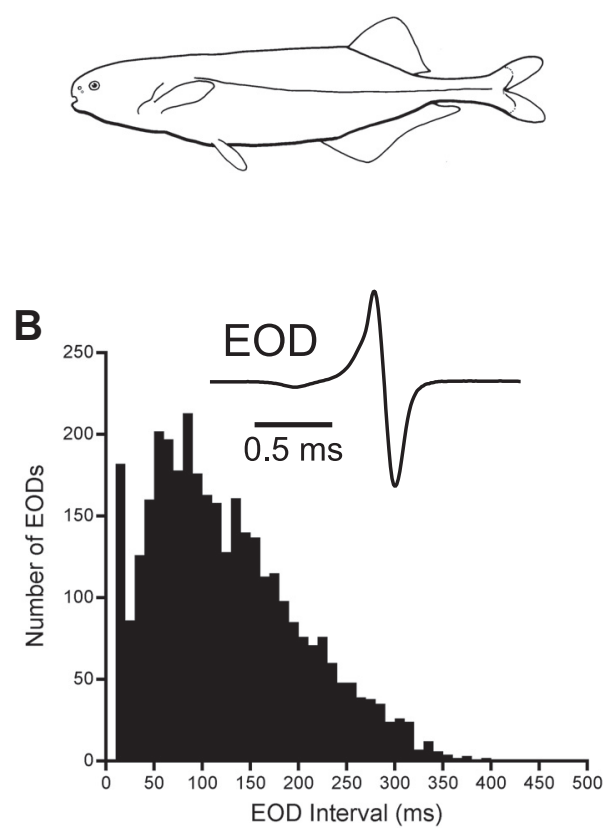

C

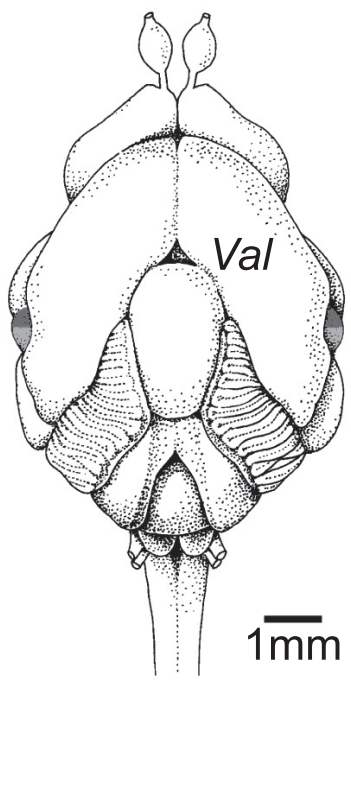

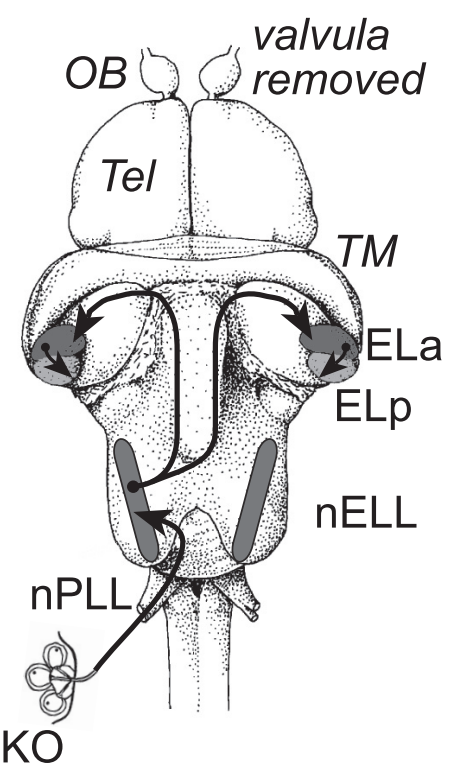

Figure 1. Electric signaling behavior and the electric communication sensory pathway in mormyrids. $A$, Outline of the mormyrid used in the study, B. brachyistius. B, Electric signaling behavior in mormyrids consists of an all-or-none EOD (inset) and the intervals between EODs, shown as a histogram of intervals from a 10 min recording of a freely behaving individual. $C$, Anatomy of the Knollenorgan electrosensory pathway. On the left is an intact brain, and on the right is a brain with the valvula cerebellum (Val) removed to expose the underlying structure. Knollenorgan (KO) primary afferents project ipsilaterally to the $n E L L$ in the hindbrain via the posterior lateral line nerve (nPLL). Neurons in the nELL project bilaterally to the ELa in the midbrain, which projects ipsilaterally to the adjacent ELp. TM, Mesencephalic tectum (optic tectum); Tel, telencephalon; OB, olfactory bulb.

that encode EODs as temporal patterns of spikes (Bennett, 1971; Zakon, 1986). Knollenorgan primary afferents project to the hindbrain nucleus of the electrosensory lateral line lobe (nELL), which relays electrosensory information to the midbrain anterior exterolateral nucleus (ELa), which in turn projects to the adjacent posterior exterolateral nucleus (ELp) (Fig. 1C). Information about the EOD waveform is first processed within ELa (XuFriedman and Hopkins, 1999), but it is unknown where information about the SPI is processed. In the current study, I used evoked potential recordings and whole-cell intracellular recordings in vivo to determine where sensitivity to the SPI emerges and to characterize the decoding of temporal patterns of stimulation by central sensory neurons.

\section{Materials and Methods}

Animals. A total of 58 Brienomyrus brachyistius (Fig. $1 A$ ), ranging in size from 7.0 to $12.0 \mathrm{~cm}$ in total length, were used in this study. They were obtained from commercial distributors who had acquired them in West Africa. The fish were housed in groups at a temperature of $25-28^{\circ} \mathrm{C}$ and conductivity of $150-300 \mu \mathrm{S} / \mathrm{cm}$ on a $12 \mathrm{~h}$ light/dark cycle and fed live black worms daily. All procedures were in accordance with the guidelines established by the National Institutes of Health and were approved by the Institutional Animal Care and Use Committees at Cornell University and Washington University in St. Louis.

Surgery. Fish were anesthetized in a solution of $300 \mathrm{mg} / \mathrm{L}$ tricaine methanesulfonate (MS-222) (Sigma-Aldrich), and then respirated under a solution of $100 \mathrm{mg} / \mathrm{L}$ MS-222 during the surgery. The fish were immobilized and electrically silenced with an intramuscular injection of flaxedil (gallamine triethiodide: $100 \mu \mathrm{l}$ of a $3 \mathrm{mg} / \mathrm{ml}$ solution; SigmaAldrich). The fish were then placed on an underwater platform and held in place with lateral supports. The platform was tilted downward toward the tail and slightly laterally so that the fish was completely immersed in water except for one side of the dorsal surface of the head. A flap of skin was removed from the head just caudal and medial to the eye, and the underlying tissue was scraped away to expose the surface of the skull.
Lidocaine (100-200 $\mu$ l of a 2\% solution; Radix Laboratories) was applied as a local anesthetic. A post was affixed to the skull using superglue to hold the fish in place and a small rectangular portion of the skull and meninges were removed to expose the surface of the midbrain exterolateral nucleus and the lateral surface of the valvula cerebellum (Fig. 1 B). A grounding/reference electrode was placed just lateral of the valvula cerebellum; the grounding electrode was then moved medially to slightly displace the valvula and expose more of the exterolateral nucleus. At the end of surgery, the respiration was switched to fresh water to bring the fish out of general anesthesia for recording.

Evoked potential recording. Evoked potentials in ELa and ELp were obtained with glass microelectrodes broken to a tip diameter of 7-12 $\mu \mathrm{m}$ and filled with $3 \mathrm{~m} \mathrm{NaCl}$. Evoked potentials were bandpass filtered from $10 \mathrm{~Hz}$ to $3 \mathrm{kHz}$ and amplified $1000 \times$ on a differential AC amplifier (A-M Systems; model 1700), and then digitized at a sampling rate of $50 \mathrm{kHz}$ (Tucker-Davis Technologies; model AD1) and saved using custom-made software for Matlab 7.4 (Mathworks).

Whole-cell intracellular recording. Whole-cell intracellular recordings from ELp neurons followed the method of Rose and Fortune (1996). Electrodes were pulled in three stages to a tip diameter of $\sim 1.2 \mu \mathrm{m}$ and filled with a tip solution containing the following (in mM): 100 potassium acetate, $2 \mathrm{KCl}, 1 \mathrm{MgCl}_{2}$, 5 EGTA, $10 \mathrm{HEPES}, 20 \mathrm{KOH}$, and 43 biocytin. The shank was filled with an identical solution except that the biocytin was replaced with mannitol. This yielded pipette resistances of 20-30 $\mathrm{M} \Omega$ and initial seal resistances $>1 \mathrm{G} \Omega$. Input resistances ranged from $\sim 120$ to $250 \mathrm{M} \Omega$.

Intracellular recordings were obtained from a total of 129 neurons in ELp that met the following criteria for inclusion: stable resting potentials of at least $-40 \mathrm{mV}$, and spikes with a height of at least $10 \mathrm{mV}$. Some neurons did not produce spikes in response to any of the stimuli presented; among these neurons, those that met the resting potential criteria and that generated spikes with a height of at least $10 \mathrm{mV}$ in response to depolarizing current injection were included.

Intracellular activity was amplified $10 \times$ on an Axopatch $200 \mathrm{~B}$ amplifier (Molecular Devices), and then digitized at a sampling rate of $50 \mathrm{kHz}$ 
A

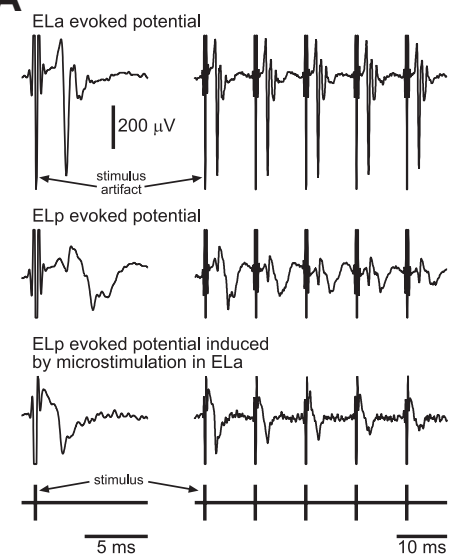

B

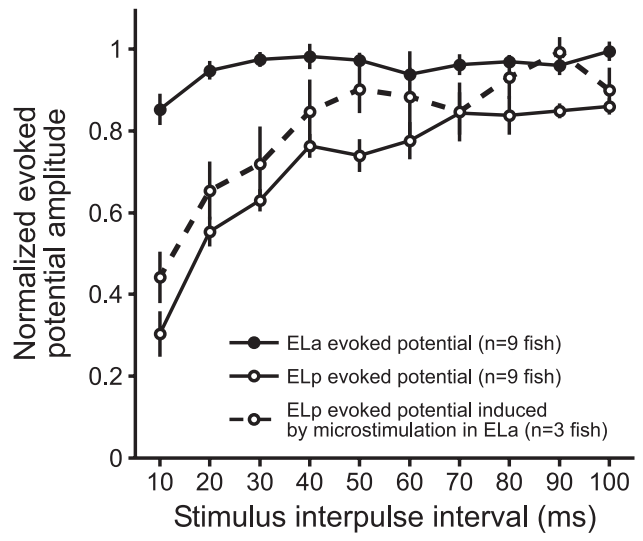

ized sequences of natural IPIs. Five to 20 repetitions were delivered for each stimulus.

Electrical microstimulation. Electrical microstimulation in ELa was used to directly drive afferent input to ELp during evoked potential and intracellular recordings from ELp. The timing of microstimulation pulses was controlled with a timing generator (Tucker-Davis Technologies; model TG6), which triggered an isolated pulse stimulator (A-M Systems; model 2100) to deliver biphasic square pulses with a duration of $100 \mu$ s and an amplitude of 15-50 $\mu \mathrm{A}$ through a concentric bipolar microelectrode (World Precision Instruments; TM33CCNON) placed on the surface of ELa.

Data analysis. A corollary discharge pathway provides inhibitory input to neurons in the hindbrain nELL, which serves to block responses in the Knollenorgan pathway to electrosensory stimuli that immediately follow electromotor output (Bell and Grant, 1989). Responses to stimulus repetitions during which an EOD command occurred within $0-5$ ms before any of the stimulus pulses were therefore excluded from analysis.

Responses to each pulse in a stimulus train were measured during a window starting im-

(Tucker-Davis Technologies; model AD1). Intracellular recordings were saved using custom-made software for Matlab 7.4.

Monitoring electromotor output. Even though the EOD is silenced by flaxedil, the EOD command can still be recorded as a three-spike potential resulting from the synchronous activation of spinal electromotor neurons (Bennett et al., 1967). Fictive electromotor output was therefore monitored by placing a pair of wires against the caudal peduncle and amplifying the signal $10,000 \times$ using a differential AC amplifier (A-M Systems; model 1700). This signal was digitized at a sampling rate of 50 $\mathrm{kHz}$ (Tucker-Davis Technologies; model AD1) and saved along with evoked potential and intracellular recording data.

Sensory stimulation. Electrosensory stimuli were generated using custom-made software for Matlab 7.4 and digital-to-analog converted at a rate of $50 \mathrm{kHz}$ (Tucker-Davis Technologies; model DA3-4). Stimulus intensity was controlled with a programmable attenuator (Tucker-Davis Technologies; model PA4), and the resulting signal was delivered to the recording chamber through an analog stimulus isolator (A-M Systems; model 2200) in either a transverse orientation, using electrodes placed on opposite sides of the fish (anodal to the left, cathodal to the right), or in a longitudinal orientation (anodal in front of the fish, cathodal behind the fish).

In most cases, biphasic square pulses were used as stimuli, although monophasic square pulses or natural EOD waveforms taken from an existing library (Carlson et al., 2000; Carlson and Hopkins, 2004) were used in some cases. For evoked potential recordings, square-wave stimuli were presented in a transverse orientation with normal polarity, at an intensity of $15-20 \mathrm{mV} / \mathrm{cm}$ and duration of $0.2-0.5 \mathrm{~ms}$. For intracellular recordings, stimulus orientation (transverse vs longitudinal), polarity (normal vs reversed), duration $(0.1-3 \mathrm{~ms})$, and intensity $(1-70 \mathrm{mV} / \mathrm{cm})$ were all systematically varied to determine the combination of attributes that elicited maximal postsynaptic potential responses to the presentation of individual pulses. Temporal-pattern sensitivity was then assessed using trains of pulses with these attributes. In many cases, a wide range of intensities elicited identical responses (Amagai, 1998); in these cases, the lowest intensity within that range was used. The observed responses to variation in pulse duration indicated that all neurons were either longpass or bandpass for pulse duration (Amagai, 1998); for long-pass neurons, the shortest pulse duration that elicited a maximal response was used; for bandpass neurons, the median pulse duration within the range eliciting a maximal response was used. A variety of temporally patterned stimuli were used to assess interpulse interval (IPI) tuning, including the following: pulse trains with constant IPIs; IPI scans, including increasing IPI scans and decreasing IPI scans; natural IPI sequences; and randommediately after a given pulse and ending immediately before the subsequent pulse (for the last pulse in a train, this window extended to the end of the recording trace). Evoked potentials were quantified by averaging the responses to each stimulus repetition and then determining the minimum potential of the evoked potential responses to each stimulus pulse. For intracellular recordings, the number of spikes per stimulus pulse was determined; to analyze membrane potentials, spikes were removed using a median filter with a width of $15 \mathrm{~ms}$ (Jagadeesh et al., 1993) and the responses to each stimulus repetition were then averaged. The resting potential was defined as the mean membrane potential during the $50 \mathrm{~ms}$ period preceding stimulation. PSP responses to each stimulus pulse were quantified as the maximum membrane potential relative to rest.

Stimulus trains consisting of 10 pulses with constant IPIs ranging from 10 to $100 \mathrm{~ms}$ were used to characterize the IPI tuning of evoked potentials and intracellular recordings. For intracellular recordings, IPI scans (either decreasing or increasing intervals ranging from 10 to $200 \mathrm{~ms}$ ) were also used in some cases. Freely behaving $B$. brachyistius typically generate IPIs ranging from $\sim 10$ to $300 \mathrm{~ms}$ (Fig. 1A) (Carlson, 2002). The range of 10-100 ms intervals was chosen because social communication displays occur at intervals well below $100 \mathrm{~ms}$ (Carlson and Hopkins, 2004; Wong and Hopkins, 2007), and both evoked potential and intracellular recordings revealed no apparent change in response as intervals were increased above $100 \mathrm{~ms}$.

Tuning curves were constructed from constant IPI trains by averaging the responses to the $2 \mathrm{nd}$ through 10 th pulses in a 10 pulse train, and then normalizing these values to the strongest response. To ensure that this measure of tuning was not distorted by transient responses to the onset of stimulus trains, several of the resulting tuning curves were compared with tuning curves based on normalized responses to either the 5th or 10 th pulses in a train. Although there were small quantitative differences between these three measures of tuning, there were no differences in the general patterns of IPI tuning. Tuning curves were constructed from IPI scans by determining the average response to each interval and then normalizing these values to the strongest response. The "best interval" (BI) was determined as the IPI that elicited the strongest response. Using a linear extrapolation between adjacent data points, the IPIs above and below the $\mathrm{BI}$ that elicited responses equal to $85 \%$ of the response at the BI (U85 and L85, respectively) were used to quantify the shape of the tuning curve. If both L85 and U85 were defined, the neuron was categorized as "bandpass"; if L85 was defined but U85 was not, the neuron was categorized as "low-pass"; if U85 was defined but L85 was not, the neuron was categorized as "high-pass"; if neither L85 nor U85 were defined, the neuron was categorized as "all-pass." For bandpass neurons, the 

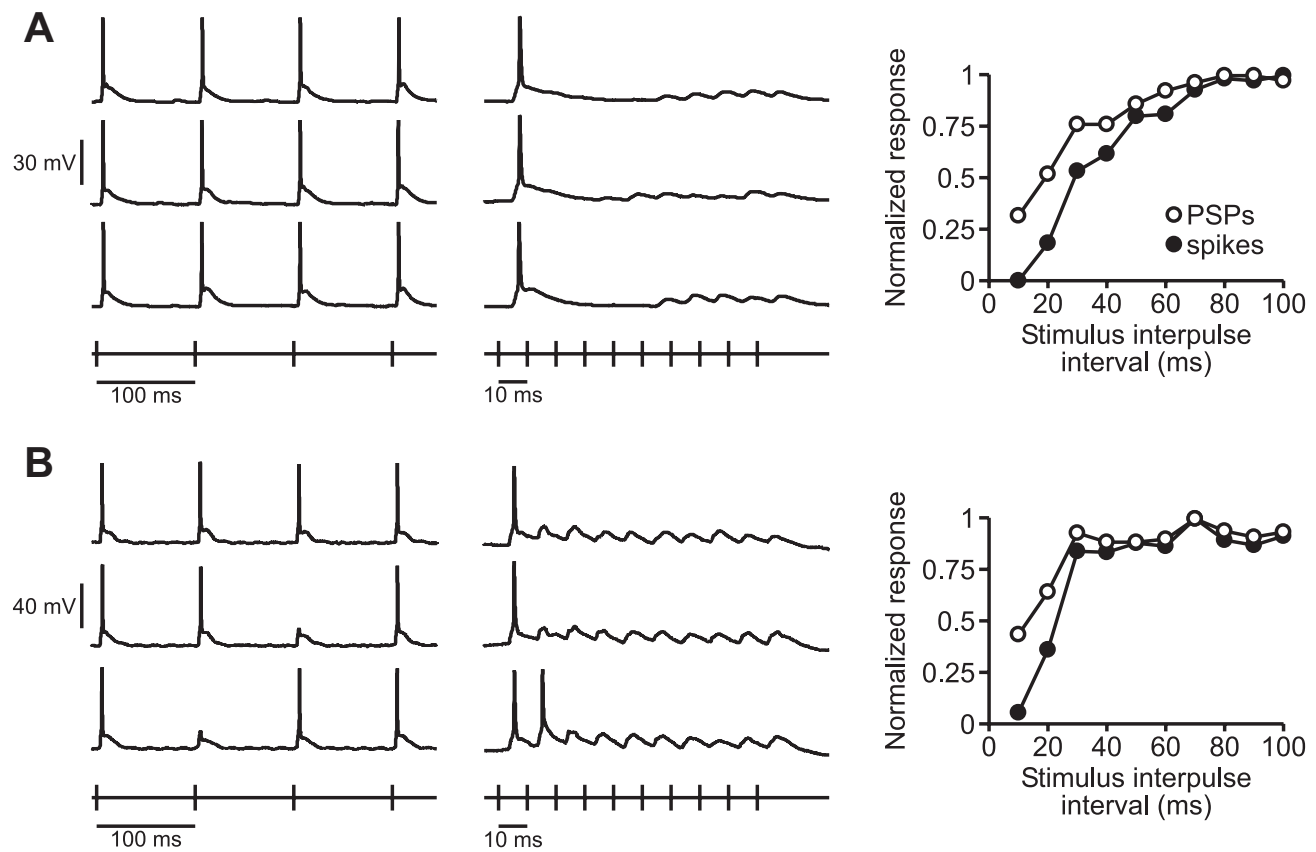

Figure 3. Intracellular recordings and tuning curves from two low-pass ELp neurons in response to constant IPI trains. $A, B$, To the left are responses to $100 \mathrm{~ms} I P I$ trains, and in the middle are responses to $10 \mathrm{~ms} I P I$ trains. The stimulus trains are shown at the bottom, and intracellular recordings from three repetitions of each stimulus are shown above. In each case, stimulus trains consisted of 10 pulses, but for clarity only the first 4 pulses are shown for the $100 \mathrm{~ms} \mathrm{IPI} \mathrm{trains.} \mathrm{To} \mathrm{the} \mathrm{right} \mathrm{are} \mathrm{normalized} \mathrm{spike} \mathrm{and} \mathrm{PSP} \mathrm{tuning} \mathrm{curves.} \mathrm{Data} \mathrm{were} \mathrm{normalized} \mathrm{by} \mathrm{averaging} \mathrm{the} \mathrm{amplitude} \mathrm{of}$ responses (either spike number or maximum PSP amplitude) to the 2nd through 10th pulses in each train, and then dividing by the maximum average response across intervals within each neuron.

bandwidth was determined as the difference between L85 and U85; for low-pass neurons, L85 was used to characterize tuning; for high-pass neurons, U85 was used to characterize tuning. For some neurons, responses to the full range of constant IPIs were not obtained; however, in some of these cases, it was possible to categorize them as low-pass, highpass, or bandpass based on characteristic response patterns to the intervals that were tested.

\section{Results}

\section{IPI tuning of evoked potential responses}

Electrosensory stimulation elicited sharp, short-latency $(\sim 2.5-$ $3.5 \mathrm{~ms}$ ) evoked potentials in ELa and broad, longer-latency ( 6-9 ms) evoked potentials in ELp (Fig. 2A), as shown in previous studies (Szabo et al., 1979; Amagai, 1998). In addition to the predominant broad, long-latency potential observed in ELp, there was typically an earlier, relatively small, sharp potential synchronous with the ELa potential (Fig. 2A). This early ELp potential has previously been interpreted as the passive spread of current from ELa (Amagai, 1998). Direct electrical stimulation of ELa using small bipolar electrodes elicited evoked potentials in ELp that were similar to the broad stimulus-evoked potentials, but with a shorter latency ( $\sim 1.5-2.5 \mathrm{~ms})$ (Fig. $2 A)$.

Evoked potentials in ELp were strongly attenuated at short IPIs, both in response to sensory stimulation and microstimulation of ELa (Fig. $2 \mathrm{~A}$ ). On average, the evoked potential responses in ELp to $10 \mathrm{~ms}$ IPIs were approximately one-half the amplitude of responses to IPIs in the range of 50-100 ms (Fig. $2 \mathrm{~B}$ ). In contrast, evoked potentials in ELa were not attenuated at short IPIs (Fig. 2A), and the normalized response magnitude was relatively constant across the range of IPIs tested (Fig. $2 B$ ). These results indicate that IPI tuning in the Knollenorgan pathway arises within ELp.

\section{IPI tuning of ELp neurons}

Whole-cell intracellular recordings were obtained from 129 ELp neurons that met the criteria for analysis (see Materials and
Methods). None of these neurons had spontaneous action potential activity, and small "bumps" in resting potential suggestive of spontaneous PSPs were only rarely observed. A total of 76 neurons were held long enough with stable resting potentials to obtain tuning curves characterizing responses to the full range of IPIs (10-100 ms). Of these 76 neurons, 53 spiked in response to at least one of the IPI trains, whereas the other 23 did not. However, these 23 neurons met the resting potential criterion for inclusion and gave strong spiking responses to depolarizing current injection. The lack of a spiking response to sensory stimulation in these neurons was most likely because they were not being adequately stimulated, either because they received input from receptors on the head that were destroyed by the surgery, or because the limited variation in stimulus geometry provided by the experimental setup did not maximally stimulate the receptive fields of some cells.

For the neurons that spiked in response to sensory stimulation $(n=53)$, there was a strong correlation between variation in spike number and variation in PSP amplitude across the range of IPIs tested (mean $r^{2} \pm$ SEM, $0.78 \pm 0.04$ ). The PSP and spike tuning curves of individual neurons had nearly identical shapes, although the spike tuning curves were typically characterized by sharper tuning, most likely because of the nonlinear effect of action potential generation (Figs. 3-5). To include all 76 neurons in the analysis, the PSP tuning curves were used for categorization. Each of the 76 neurons with a full tuning curve was thereby categorized as low-pass, high-pass, bandpass, or all-pass based on whether the response dropped below $85 \%$ of the maximum response at intervals longer and shorter than the BI (see Materials and Methods). This resulted in 33 low-pass neurons $(43.4 \%)$ (Fig. 3), 21 high-pass neurons (27.6\%) (Fig. 4), 18 bandpass neurons $(23.7 \%)$ (Fig. 5), and 4 all-pass neurons (5.3\%).

The $85 \%$ of maximum response criterion was used to define the low cutoff intervals of low-pass neurons, the high cutoff intervals of high-pass neurons, and both low and high cutoff 
A

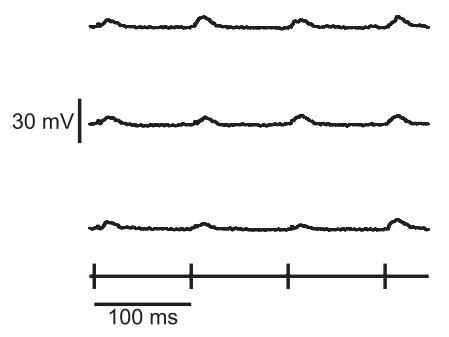

B

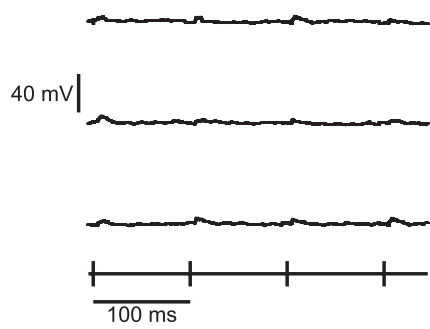

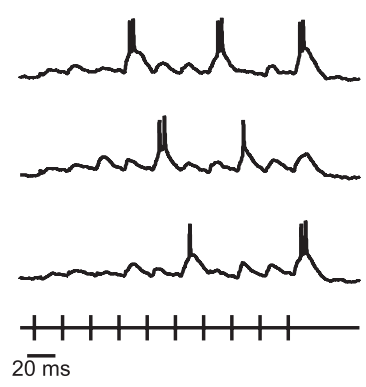
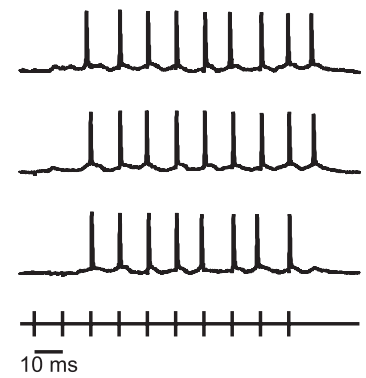
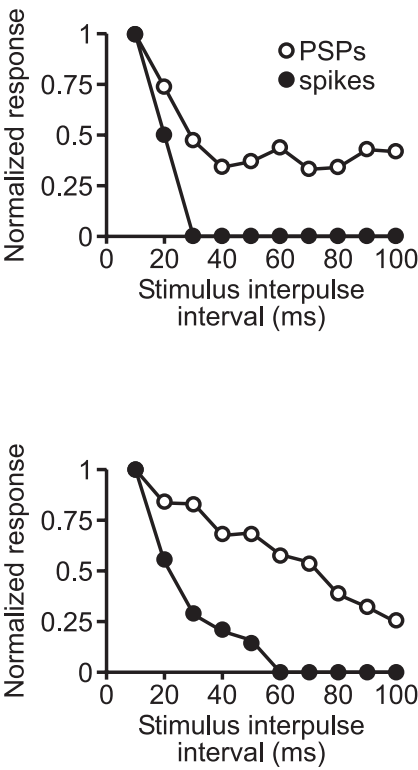

Figure 4. Intracellular recordings and tuning curves from two high-pass ELp neurons in response to constant IPI trains, presented as in Figure 3. To the left are responses to 100 ms IPI trains, and in the middle are responses to 10 or 20 ms IPI trains. The stimulus trains are shown at the bottom, and intracellular recordings from three repetitions of each stimulus are shown above. In each case, stimulus trains consisted of 10 pulses, but for clarity only the first 4 pulses are shown for the $100 \mathrm{~ms}$ IPI trains. To the right are normalized spike and PSP tuning curves.
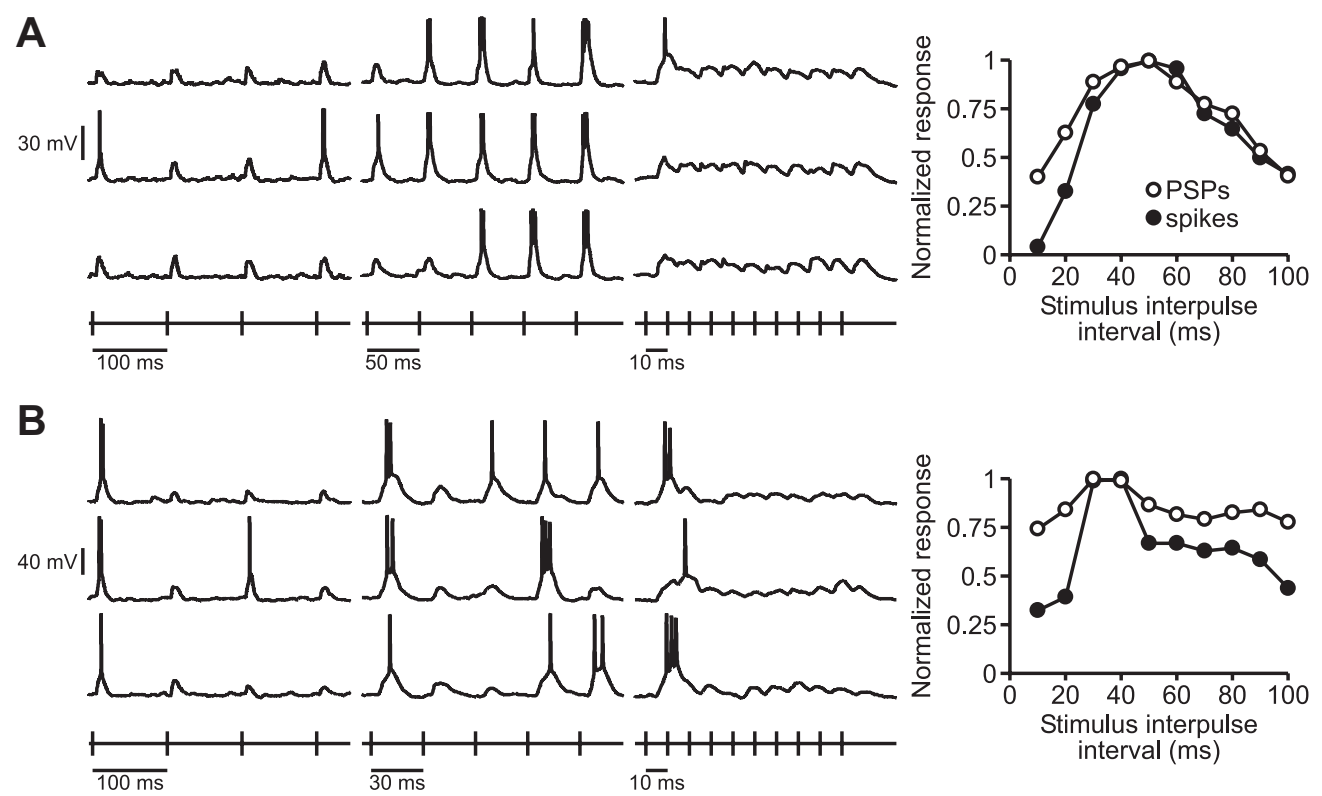

Figure 5. Intracellular recordings and tuning curves from two bandpass ELp neurons in response to constant IPI trains, presented as in Figure 3. $A$, Responses of a bandpass ELp neuron to a 100 $\mathrm{ms} I P I$ train (left), $50 \mathrm{~ms} I P \mathrm{I}$ train (the BI; second from the left), and $10 \mathrm{~ms} I \mathrm{PI}$ train (third from the left), with the normalized spike and PSP tuning curves (right). In each case, stimulus trains consisted of 10 pulses, but for clarity only the first 4 and 5 pulses are shown for the 100 and $50 \mathrm{~ms} I P I$ trains, respectively. $\boldsymbol{B}$, Responses of a bandpass ELp neuron to a $100 \mathrm{~ms} I P I$ train (left), $30 \mathrm{~ms} I P I$ train (the $\mathrm{Bl}$; second from the left), and $10 \mathrm{~ms}$ IPI train (third from the left), with the normalized spike and PSP tuning curves (right). In each case, stimulus trains consisted of 10 pulses, but for clarity only the first 4 and 5 pulses are shown for the 100 and $30 \mathrm{~ms}$ IPI trains, respectively.

intervals of bandpass neurons (see Materials and Methods). This revealed extensive variation in the shapes of the tuning curves for all three categories. The low cutoff intervals of low-pass neurons ranged from 16.71 to $92.98 \mathrm{~ms}$ (Fig. 6). The high cutoff intervals of high-pass neurons ranged from 10.15 to $91.33 \mathrm{~ms}$ (Fig. 6). Bandpass neurons exhibited widespread variation in BI, which varied over all possible values, from 20 to $90 \mathrm{~ms}$, although the vast majority ( 13 of 18 , or $72.0 \%$ ) was in the range of $30-70 \mathrm{~ms}$ (Fig. 6). The bandwidth of bandpass neurons (difference between high and low cutoff values) ranged from 8.70 to $84.69 \mathrm{~ms}$ (Fig. 6). Among bandpass neurons, bandwidth was correlated with BI (i.e., neurons tuned to longer intervals tended to have broader tuning curves; $r^{2}=0.31, p<0.02$ ).

It is possible than some neurons categorized as low-pass would actually be categorized as bandpass if longer intervals had been tested; however, this would probably only be true for a small number of neurons given that all but one of the neurons categorized as bandpass (94.4\%) had BIs <90 ms (Fig. 6). In addition, 


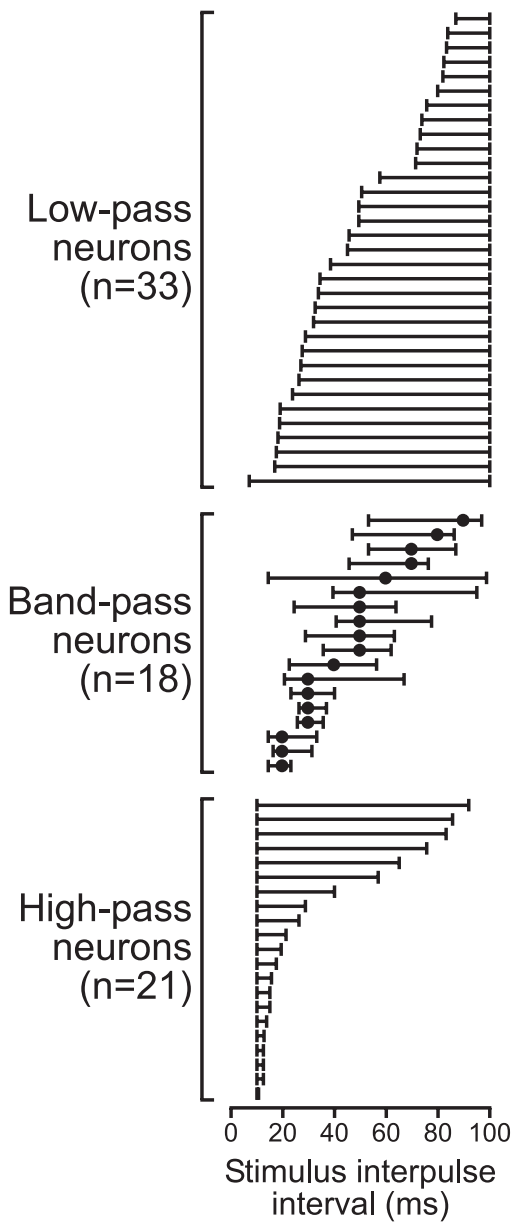

Figure 6. Tuning of ELp neurons to constant IPI trains. For bandpass neurons, the BI (the interval that elicited the strongest PSP response) is shown as a circle, and the bars extend to the lower and upper intervals at which the response dropped to $85 \%$ of the response at the BI, based on linear extrapolation between adjacent data points. Data are organized from bottom to top first in order of increasing Bl and second in order of increasing bandwidth. For low-pass and high-pass neurons, the full range of responses greater than the $85 \%$ of maximum response are shown. For high-pass neurons, data are organized from bottom to top in order of increasing bandwidth. For low-pass neurons, data are organized from bottom to top in order of decreasing bandwidth.

there was no apparent difference in the responses of low-pass neurons to pulses during $100 \mathrm{~ms}$ IPI trains and individual pulses presented at rates $<1 / \mathrm{s}$ (data not shown), and low-pass neurons responded equally strongly to the onset of short IPI trains (Fig. 3). Therefore, low-pass neurons appeared to respond equally to all pulse intervals exceeding a critical duration. It is also possible that some neurons categorized as high-pass may have been categorized as bandpass if shorter intervals had been tested, but the study species ( $B$. brachyistius) never generates intervals $<8 \mathrm{~ms}$ (Carlson, 2002; Carlson and Hopkins, 2004; Wong and Hopkins, 2007), so such tuning, if it existed, would not be behaviorally relevant.

\section{Rate-dependent modulation of postsynaptic potentials in ELp neurons}

Low-pass neurons responded to long IPIs with robust, depolarizing PSPs well above threshold for action potential generation (Fig. 3). At short IPIs, however, these PSPs were strongly attenuated throughout the course of the stimulus train, thereby eliminating or severely reducing the amount of spiking (Fig. 3). For all low-pass neurons, the amount of attenuation was greatest for the shortest intervals tested $(10-20 \mathrm{~ms})$, and as stimulus interval was increased, there was a progressive reduction in the amount of attenuation (Fig. 7A). However, low-pass neurons differed among each other in the range of intervals that elicited PSP attenuation, and these differences were responsible for the variation in IPI tuning ranges seen in Figure 6. For example, the neuron in Figure $7 A i$ was characterized by PSP attenuation in response to IPIs as long as $60 \mathrm{~ms}$, resulting in a low cutoff interval of 72.89 ms. In contrast, PSPs of the neuron in Figure 7Aii were only attenuated at IPIs of 10 and $20 \mathrm{~ms}$, resulting in long-pass tuning that extended to a low cutoff interval of $27.23 \mathrm{~ms}$. The neuron in Figure 7Aiii was intermediate, with PSP attenuation evident at IPIs of up to $40 \mathrm{~ms}$ and a low cutoff interval of 45.49 ms. In some cases, there was a clear hyperpolarization evident after the end of short IPI trains in low-pass neurons (Fig. 7Aii).

High-pass neurons responded to long IPIs with relatively weak, subthreshold PSPs (Fig. 4). In response to short IPIs, however, these potentials were enhanced during the stimulus train, often reaching threshold for action potential generation (Fig. 4). The responses of several high-pass neurons consisted of a combination of depolarizing and hyperpolarizing potentials. For example, the responses of the neuron in Figure $7 \mathrm{Bi}$ to long IPIs and the onset of short IPI trains were dominated by hyperpolarization; throughout the course of short IPI trains, however, there was a gradual switch from hyperpolarizing to depolarizing responses, resulting in stronger responses to short IPIs. Similarly, the neuron in Figure 7 Bii responded to long IPIs and the onset of short IPI trains with a complex mix of depolarization followed by hyperpolarization; throughout the course of short IPI trains, however, the responses became dominated by depolarization, resulting in stronger responses to short IPIs. In other cases, highpass neurons responded with depolarizations only, and tuning to short intervals resulted from an increase in the amplitude of these depolarizations (Fig. 7Biii). In nearly every case, however, hyperpolarizations were evident in high-pass neurons at the end of short IPI trains (Fig. 7Bi-Biii).

Among all of the high-pass neurons, the enhancement of PSP responses was greatest for the shortest intervals tested (10-20 $\mathrm{ms}$ ), and as stimulus interval was increased, there was a progressive reduction in the amount of enhancement (Fig. 7B). Similar to low-pass neurons, however, high-pass neurons differed among each other in the range of intervals that elicited enhancement, and these differences established the variation in IPI tuning ranges seen in Figure 6. For example, the neuron in Figure 7Bii had PSPs that were enhanced at IPIs as long as $80 \mathrm{~ms}$, resulting in relatively broad tuning up to a high cutoff interval of $85.05 \mathrm{~ms}$. In contrast, the neurons in Figure 7, Bi and Biii, responded with enhanced PSPs at only the shortest IPIs tested (10-20 ms), resulting in much narrower high-pass tuning with high cutoff intervals of 15.38 and $26.21 \mathrm{~ms}$, respectively.

The IPI tuning of bandpass neurons typically resulted from PSPs that were enhanced in response to intermediate IPIs (Figs. $5 A, 7 C)$. For many bandpass neurons, short IPI trains resulted in attenuating PSPs similar to those observed in low-pass neurons, which served to sharpen IPI tuning (Figs. 5A,B,7Ciii), whereas in other bandpass neurons no such attenuation was observed (Fig. $7 \mathrm{Ci}, \mathrm{Cii})$. In rare cases, there was no apparent enhancement of responses to intermediate IPIs and bandpass tuning resulted solely from PSP attenuation that was strongest in response to both long and short IPIs (Fig. 5B). In some cases, there was a clear hyperpolarization evident in bandpass neurons during stimulus trains (Fig. 7Ci,Ciii). 


\section{low-pass neurons}

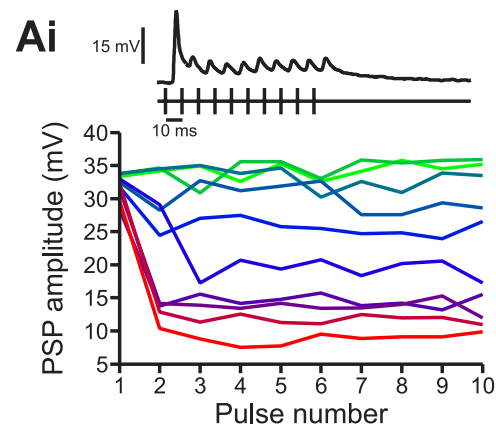

high-pass neurons

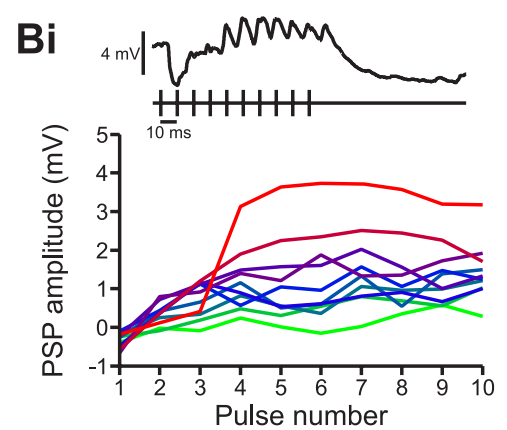

band-pass neurons

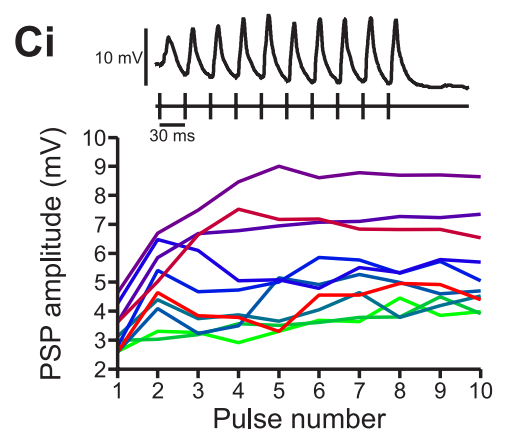

stimulus interpulse interval (ms)
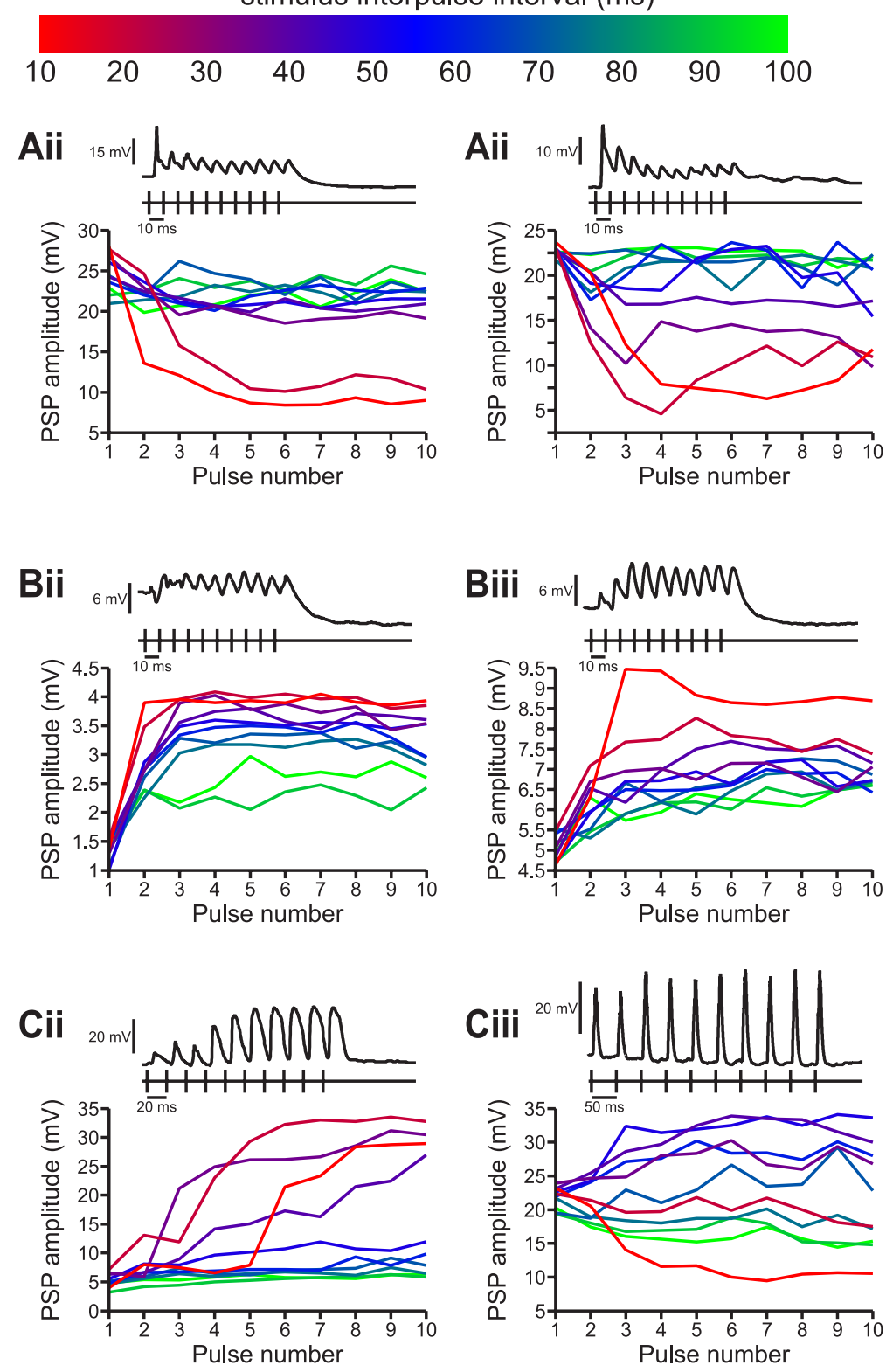

Figure 7. Rate dependence of PSP responses in ELp neurons. $\boldsymbol{A}$, Three low-pass neurons. $\boldsymbol{B}$, Three high-pass neurons. $\boldsymbol{C}$, Three bandpass neurons. The recording traces from each neuron show the average membrane potential across stimulus repetitions after median filtering in response to $10 \mathrm{~ms} I P I$ trains for the low-pass and high-pass neurons, and in response to the BI train for the bandpass neurons (stimuli are shown beneath each trace). The plots show PSP amplitude as a function of pulse number for IPIs ranging from 10 to $100 \mathrm{~ms}$.

To confirm the evoked potential result indicating that IPI tuning arises within local ELp circuitry rather than earlier in the sensory pathway (Fig. 2), electrical microstimulation on the surface of ELa was used to directly stimulate afferent axons to ELp. Individual neurons responded slightly differently to natural sensory stimulation and direct stimulation of ELa afferents (Fig. 8), most likely because of the activation of different populations of afferents by the two methods. Nevertheless, the general pattern of IPI tuning is consistent for both types of stimulation, with lowpass neurons responding with attenuating responses at short intervals (Fig. 8A) and high-pass neurons responding with enhanced responses at short intervals (Fig. $8 B$ ). For several ELp neurons within each category, square wave depolarizing or hyperpolarizing current pulses were injected at IPIs of 10-100 ms, and this resulted in consistent voltage shifts in response to each pulse regardless of IPI (data not shown). This indicates that the passive filtering properties of ELp neurons (i.e., capacitance) did not contribute to IPI tuning. However, this does not rule out intrinsic, voltage-dependent conductances contributing to PSP enhancement or attenuation.

\section{Tuning of ELp neurons to dynamic changes in IPI}

To characterize tuning to the time course of IPI changes, stimuli with smoothly increasing and decreasing IPIs ranging from 10 to $200 \mathrm{~ms}$ were presented, and PSP amplitudes in response to the same intervals during increasing or decreasing IPI scans were compared. Some neurons showed nearly identical responses to increasing and decreasing IPIs (Fig. 9Ai, low-pass neuron; Bi, highpass neuron; $C i$, bandpass neuron). However, others showed different responses to the same intervals depending on whether they occurred while IPIs were increasing or decreasing, evident as hysteresis when comparing the increasing and decreasing IPI tuning 
curves. For example, the low-pass neurons in Figure 9, Aii and Aiii, showed stronger tuning during increasing IPI scans than decreasing IPI scans; this was attributable to more strongly attenuated responses to short IPIs during an increasing IPI scan for the neuron in Figure 9Aii, but more strongly attenuated responses to long IPIs during a decreasing IPI scan for the neuron in Figure 9Aiii. The high-pass neuron in Figure 9Bii shows little tuning to IPI during an increasing IPI scan, but relatively strong tuning to IPI during a decreasing IPI scan. Similarly, the high-pass neuron in Figure 9Biii responded more strongly to decreasing IPIs than increasing IPIs, resulting in especially strong tuning during a decreasing IPI scan. The bandpass neuron in Figure 9Cii showed little tuning during a decreasing IPI scan, but was strongly tuned to 30 ms IPIs during an increasing IPI scan.

The amount of hysteresis for each neuron was quantified by measuring the magnitude of the difference in PSP amplitude for each interval during increasing versus decreasing IPI scans, summing across all intervals, and then dividing by the maximum PSP amplitude and total number of intervals. This generated a normalized measure of hysteresis that varies from 0 (no hysteresis) to 1 (maximum hysteresis, wherein all responses are maximal in one direction and 0 in the other). Across all neurons tested $(n=42)$, the amount of hysteresis varied from 0.04 to 0.77 . However, there were no significant differences among low-pass, bandpass, and high-pass neurons (ANOVA: $F_{(2,36)}=1.96 ; p=$ $0.16)$, and there was no relationship between hysteresis and $\mathrm{BI}$ (Spearman's rank $r=-0.13 ; p=0.41$ ). Therefore, tuning to the direction of change in stimulus interval appears to be an additional characteristic of ELp neuron tuning that varies independent of tuning to interval.

\section{The detection of social communication displays through temporal pattern recognition}

The social communication displays of $B$. brachyistius consist of a large repertoire of different SPI patterns (Carlson, 2002; Wong and Hopkins, 2007). Three quantitatively distinct modal categories of burst displays have been described previously (Carlson and Hopkins, 2004): (1) scallops, which are a brief, stereotyped SPI consisting of decreases in interval to values of 8-15 ms; (2) accelerations, which are a graded display consisting of moderate, sustained, variable duration decreases in interval to values of 20-60 $\mathrm{ms}$; and (3) rasps, which are a combination of a scallop followed by an acceleration. The tuning of ELp neurons to IPI and the direction of change in interval suggests that, in the context of natural signaling behavior, different ELp neurons may be specialized for the detection of different types of displays. To test this hypothesis, I stimulated fish with natural SPIs containing either a scallop, acceleration, or rasp recorded from freely behaving fish, available from a preexisting library of SPI recordings (Carlson and Hopkins, 2004).

The response of a typical high-pass neuron to all three patterns is shown in Figure $10 \mathrm{~A}$. High-pass neurons typically responded to the long, "resting" intervals occurring before and after these displays with relatively weak potentials that did not elicit spiking. During scallops and the early phase of rasps, these potentials were strongly enhanced, resulting in consistent spiking responses. During accelerations and the late phase of rasps, however, there was only a modest increase in PSP amplitude, if any, and either no spiking or sporadic, infrequent spiking.

The response of a typical bandpass neuron to all three patterns is shown in Figure $10 \mathrm{~B}$. Bandpass neurons were also typically silent during the long, resting intervals occurring before and after these displays. During scallops and the early phase of rasps, there were only small increase in PSP amplitude, if any, and no spiking. During accelerations and the late phase of rasps, however, there was a relatively strong increase in PSP amplitude and consistent spiking.

The responses of low-pass neurons to these displays was essentially the opposite of high-pass neuron responses (Fig. 10C). Low-pass neurons consistently spiked in response to the slow resting intervals preceding and following the displays. During scallops and the early phase of rasps, however, the PSPs were strongly attenuated, resulting in a complete cessation of spiking. During accelerations and the late phase of rasps, there was a relatively modest decrease in PSP amplitude and spiking; for the neuron shown in Figure 10C, this decrease only occurred during the early portion of these displays and the response recovered before the acceleration (or late phase of the rasp) ended.

Although accelerations exhibit continuous, graded variation in their duration and SPI pattern, scallops are characterized by a highly stereotyped SPI. A detailed analysis of scallop responses was therefore performed to determine which combination of IPI tuning and hysteresis (i.e., tuning to direction of change in interval) is best for establishing scallop detection by single neurons. Responses to scallops were quantified by subtracting the maximum PSP amplitude during the resting period before the scallop from the maximum PSP amplitude during the scallop. High-pass neurons showed a significant increase in PSP amplitude in response to scallops (mean change in PSP amplitude \pm SEM $=$ $\left.4.83 \pm 1.28 ; t_{(9)}=3.78 ; p<0.005\right)$, whereas bandpass neurons showed no significant change in PSP amplitude (1.14 \pm 2.12 ; $\left.t_{(6)}=1.14 ; p=0.61\right)$, and low-pass neurons showed a significant decrease in PSP amplitude $\left(-9.64 \pm 3.71 ; t_{(6)}=-2.60 ; p<\right.$ $0.05)$. Accordingly, there was a significant negative correlation 
Ai

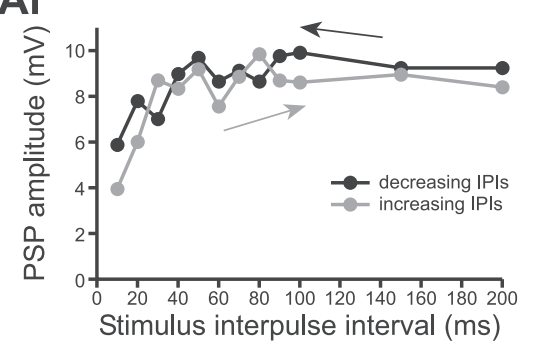

Aii

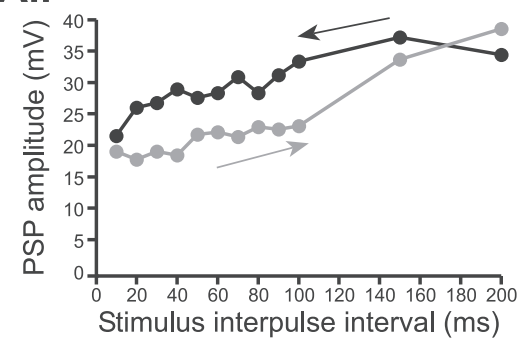

Aiii

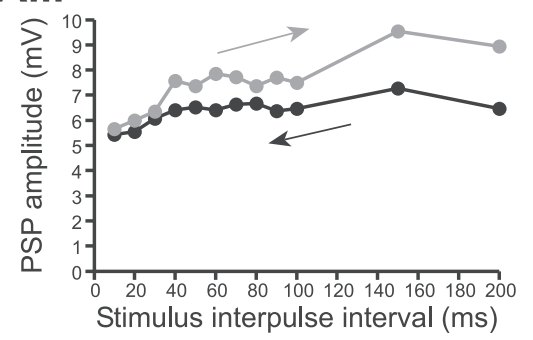

Ci

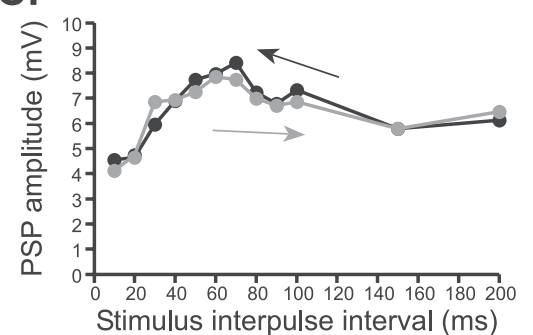

$\mathrm{Bi}$

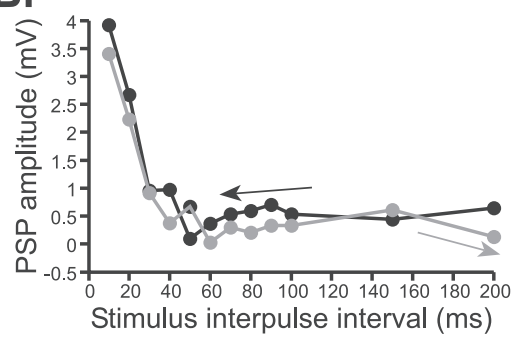

\section{Bii}

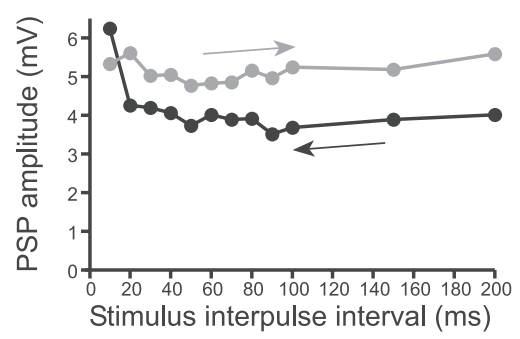

Biii

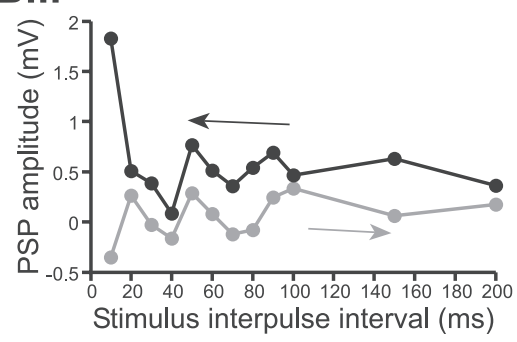

\section{Cii}

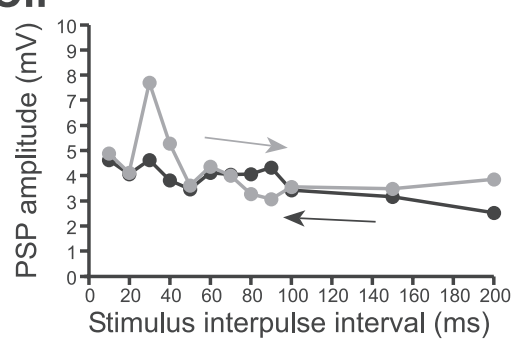

To test the hypothesis that the responses of low- and high-pass neurons to scallops are influenced not only by IPI but by the specific sequence of IPIs that characterize the display, responses to natural SPIs containing scallops were compared with the responses to randomized sequences of the same IPIs. For high-pass neurons, there was a much stronger increase in spiking activity in response to the natural sequences (Fig. 11A). Similarly, low-pass neurons responded with a stronger, more consistent decrease in spiking in response to the natural sequences (Fig. $11 B)$. This suggests that the temporal dynamics of PSP responses in these neurons results in selective tuning to social communication displays characterized by stereotyped IPI sequences.

\section{Discussion}

The temporal pattern of signaling plays a central role in the social behavior of mormyrids (Carlson, 2002). A variety of electrical displays are generated in specific social contexts, including courtship and mating (Hopkins and Bass, 1981; Wong and Hopkins, 2007), social organization (Moller, 1976; Moller et al., 1979; Arnegard and Carlson, 2005), aggression and territoriality (Bell et al., 1974; Kramer, 1974, 1976; Kramer and Bauer, 1976), and exploration and feeding (von der Emde, 1992; Arnegard and Carlson, 2005). The initial decoding of the EOD waveform occurs within the first midbrain stage of electrosensory processing, the ELa (XuFriedman and Hopkins, 1999). In the current study, evoked potential recordings indicate that the initial processing of the SPI occurs in the ELp, the sole target of ELa output neurons (Fig. 2). The vast majority of ELp neurons were tuned to IPI (Figs. 3-6), and the tuning of evoked potentials and single neurons showed the same pattern for both sensory stimbetween BI and scallop responses ( $n=24$; Spearman's rank $r=$ $-0.67 ; p<0.001$ ); neurons tuned to short intervals tended to respond to scallops with significant increases in PSP amplitude, whereas neurons tuned to long intervals tended to respond to scallops with significant decreases in PSP amplitude. Tuning to the direction of change in interval also appeared to play an important role in sensitivity to scallops, as there was a significant positive correlation between hysteresis and scallop responses $(n=10$; Spearman's rank $r=0.95 ; p<0.0001)$. However, there was no significant difference in scallop responses between neurons that exhibited stronger responses to increasing IPIs versus neurons that exhibited stronger responses to decreasing IPIs $\left(t_{(8)}=0.98 ; p=0.36\right)$. Therefore, neurons tuned to short intervals that were also sensitive to either increases or decreases in interval were ideally suited to detecting scallops. This makes sense, given that scallops are characterized by rapid decreases in IPI to short intervals followed by rapid increases in IPI.

ulation and direct electrical stimulation of ELa afferent axons (Figs. 2, 8).

Three distinct patterns of IPI tuning were observed among ELp neurons in B. brachyistius: low-pass neurons tuned to long intervals (Fig. 3), high-pass neurons tuned to short intervals (Fig. 4), and bandpass neurons tuned to intermediate intervals (Fig. 5). Many ELp neurons were also sensitive to the direction of change in interval (Fig. 9). The classification of neurons based on IPI tuning was based on arbitrary criteria; these three classes may not represent distinct categories, but simply variation along a continuum. Regardless, it is clear that ELp neurons exhibit broad diversity in IPI tuning (Fig. 6) that matches the wide range of intervals generated by freely behaving fish (Fig. 1). In fact, the three categories of tuning are well suited to representing the different modal categories of communication displays in B. brachyistius (Carlson and Hopkins, 2004), with low-pass neurons representing resting activity, high-pass neurons representing "scallops," 
A

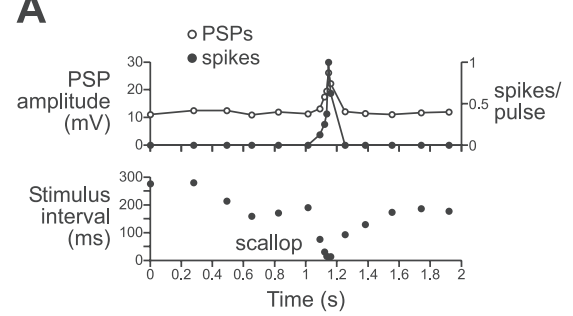

B

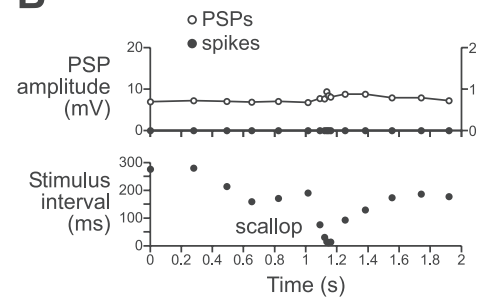

C
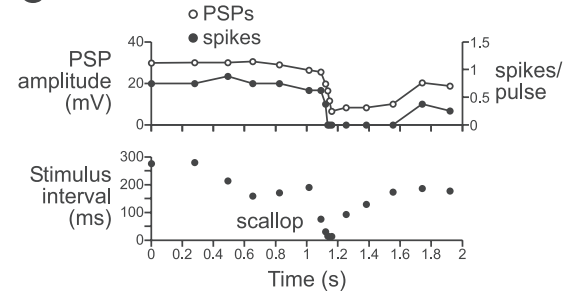

\section{high-pass neuron}
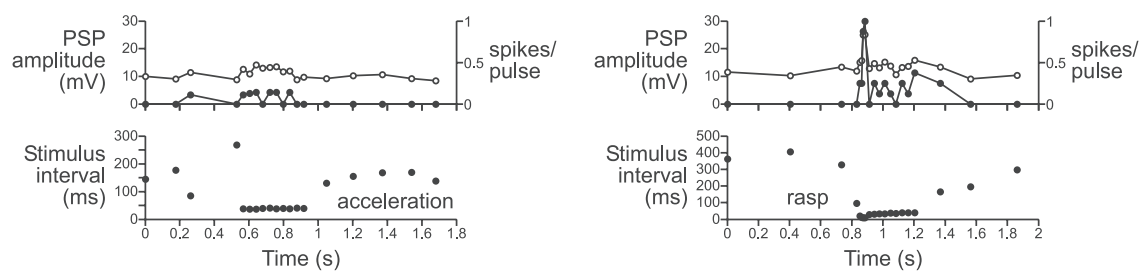

band-pass neuron
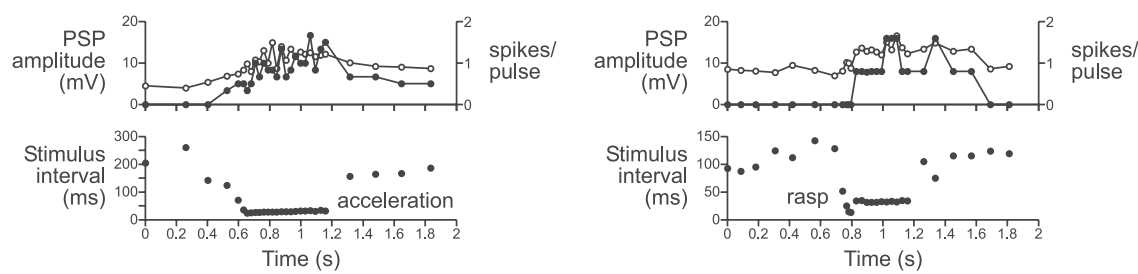

low-pass neuron

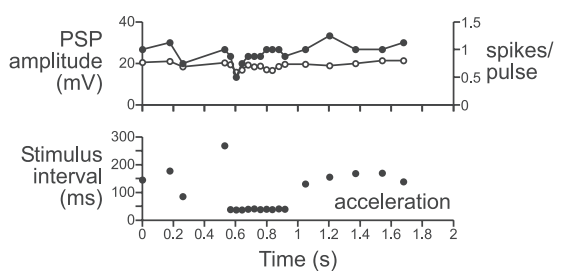

Figure 10. Responses of a high-pass neuron $(\boldsymbol{A})$, bandpass neuron $(\boldsymbol{B})$, and low-pass neuron $(\boldsymbol{C})$ to stimulus patterns containing a scallop (left), acceleration (middle), or rasp (right), all recorded from freely behaving animals. In each case, the bottom plot shows the SPI, and the top plot shows both PSP amplitude and the average number of spikes per stimulus pulse.

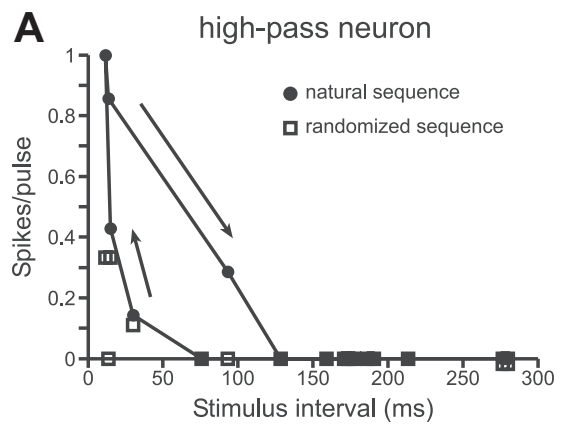

B

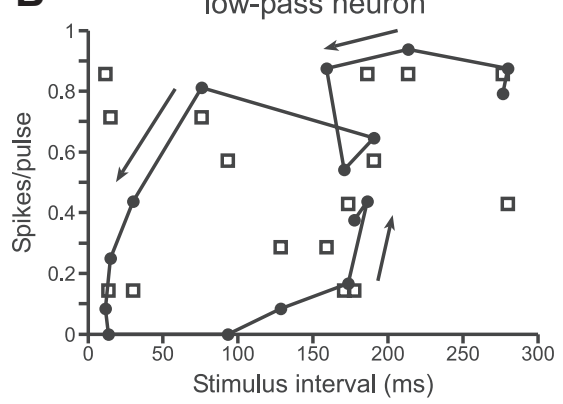

Figure 11. Effects of randomizing the natural SPI on spiking responses to SPIs containing a scallop in a high-pass neuron $(\boldsymbol{A})$ and a low-pass neuron $(\boldsymbol{B})$. For the natural SPI, the arrows show the direction of change in interval.

and bandpass neurons representing "accelerations" (Fig. 10). Even if the categorization of neurons is artificial, it is clear that the tuning of individual neurons to IPI as well as dynamic changes in IPI create "labeled lines" for specific social communication dis- plays. This conclusion is strengthened by the observation that randomizing the stereotyped SPI that characterizes scallops results in smaller increases and decreases in the firing rates of high-pass and low-pass neurons, respectively (Fig. 11). Differences in tuning among high-pass neurons may allow fish to discriminate the different SPI patterns that characterize the scallops of different individuals (Carlson and Hopkins, 2004), and differences in tuning among bandpass neurons may allow fish to discriminate among accelerations, which exhibit wide variation in IPI both within and between individuals (Carlson and Hopkins, 2004; Wong and Hopkins, 2007). Neurons at later stages of the pathway may extract information about display duration by comparing the timing of inputs from neurons that prefer increasing or decreasing IPIs.

The IPI tuning of ELp neurons was directly related to ratedependent changes in PSP amplitude (Fig. 7). Low-pass neurons responded to short IPIs with PSP attenuation (Figs. 3, 7A), whereas high-pass neurons responded to short IPIs with PSP enhancement (Figs. 4,7B). Bandpass neurons were more variable in their response patterns, but a typical feature was the enhancement of PSP amplitude over intermediate IPIs (Figs. 5, 7C). In some cases, tuning to intermediate IPIs was established or enhanced by PSP attenuation in response to short and/or long IPIs. These rate-dependent changes in PSP amplitude suggest a role for short-term synaptic plasticity in establishing IPI tuning (Zucker and Regehr, 2002). In vitro brain slice studies reveal that shortterm synaptic plasticity is an important mechanism for the filtering of presynaptic spike trains (Abbott and Regehr, 2004), and short-term synaptic plasticity is increasingly being recognized as a general mechanism for generating temporal filters for information processing in vivo (Fortune and Rose, 2001). In terms of 
decoding specific spike patterns, a computational modeling study revealed that tuning to presynaptic IPIs could result from the integration of excitatory and inhibitory inputs that differ in their short-term synaptic plasticity (Buonomano, 2000). A recent study in the auditory system of frogs revealed that bandpass tuning to sound pulse interval results from a combination of nonplastic inhibition and excitation that is enhanced over a specific range of intervals (Edwards et al., 2007). Together with the current study, this supports a role for short-term synaptic plasticity in establishing tuning to presynaptic interspike interval.

The responses of several neurons within all three classes were indicative of a combination of excitatory and inhibitory synaptic input. In some cases, this was evident as a hyperpolarization at stimulus offset (Fig. 7Aii,Bi-Biii,Ci,Ciii); in other cases, this was evident as an alternating pattern of depolarization and hyperpolarization that developed throughout the course of a stimulus train (Fig. 7Ci); finally, many high-pass neurons responded to stimulus onset with hyperpolarizations that attenuated throughout the course of short IPI trains (Fig. 7Bi,Bii).

Several different patterns of excitatory and inhibitory shortterm plasticity could account for these rate-dependent changes in PSPs. The attenuation of PSP responses at short IPIs for low-pass neurons could result from depression in excitatory pathways, facilitation in inhibitory pathways, or both. In frogs, a subset of low-pass auditory neurons responds similarly to short IPI trains: a phasic response to train onset that attenuates throughout the course of the train, resulting from either delayed inhibition or depression at excitatory synapses (Edwards et al., 2008). Conversely, the enhancement of PSP responses at short IPIs for high-pass neurons could result from facilitation in excitatory pathways, depression in inhibitory pathways, or both. These types of responses could be attributable to plasticity at local synapses onto a particular neuron, or they could be attributable to plasticity at upstream synapses on other ELp neurons. Indeed, it is highly likely that network interactions among ELp neurons play an important role in establishing IPI tuning. For example, low-pass and high-pass neurons may provide mutual inhibition. The enhancement of PSP responses at intermediate IPIs in bandpass neurons could be attributable to rate-dependent facilitation of excitatory input over a limited IPI range as found in the auditory system of frogs (Edwards et al., 2007), coincident excitatory input from low-pass and high-pass neurons with overlapping tuning ranges, or inhibitory input from low-pass neurons at long IPIs and inhibitory input from high-pass neurons at short IPIs. The diversity of bandpass neuron response patterns suggests that several mechanisms may be involved.

It remains unclear how IPI tuning relates to the encoding of other stimulus features, such as pulse duration, amplitude, and polarity (Amagai, 1998). In the current study, the combination of these variables that elicited the maximal response was used; in the future, it will be interesting to determine whether there is a relationship between tuning to pulse attributes and IPI, and whether patterns of IPI tuning remain consistent for pulses with different attributes. If different pulses elicit different complements of excitation and inhibition, then this could provide an experimental tool to assess the contributions of excitatory and inhibitory pathways in establishing IPI tuning.

Determining the importance of network interactions, shortterm synaptic plasticity in excitatory and inhibitory pathways, and the exact location of plastic changes in synaptic transmission will involve a combination of anatomical studies to characterize the morphology and projection patterns of ELp neurons, immunohistochemistry to localize neurotransmitter receptor types, in vivo electrophysiology using current clamp to estimate excitatory and inhibitory synaptic conductances, and in vitro electrophysiology using voltage clamp, focal stimulation, and pharmacological manipulation. Although in vitro studies have yielded significant insight into synaptic physiology, a major limitation is that the relationship between the stimulation patterns used and the types of presynaptic spike trains that occur in vivo is unclear. Furthermore, because of the complexity of these circuits and the information they process, there is an imprecisely defined relationship between stimulation patterns and information processing. In contrast, in vivo studies allow one to directly assess the role of a neuron in sensory processing, but generally suffer from a lack of experimental control. The mormyrid ELp circuit overcomes many of the limitations of both approaches. When stimulus waveform is kept constant, the timing of presynaptic input from ELa directly follows the pattern of sensory stimulation; this provides a unique opportunity to directly control the timing of presynaptic input and thereby study the processing of behaviorally relevant patterns of activity in an intact brain. Furthermore, the diversity of social signals used by mormyrids means that the circuit must process a wide range of temporal patterns. Finally, as the microstimulation results make clear (Figs. 2, 7), an in vitro brain slice preparation containing ELa and ELp will allow for the generation of the same patterns of presynaptic input for direct comparison with in vivo results. Research on temporal processing in ELp should therefore help bridge in vivo studies of information processing with in vitro studies of cellular and synaptic mechanisms to yield significant insight into the synaptic and network mechanisms for temporal-pattern decoding and its role in information processing.

\section{References}

Abbott LF, Regehr WG (2004) Synaptic computation. Nature 431:796-803. Amagai S (1998) Time coding in the midbrain of mormyrid electric fish. II. Stimulus selectivity in the nucleus exterolateralis pars posterior. J Comp Physiol A Neuroethol Sens Neural Behav Physiol 182:131-143.

Arnegard ME, Carlson BA (2005) Electric organ discharge patterns during group hunting by a mormyrid fish. Proc Biol Sci 272:1305-1314.

Bell CC, Grant K (1989) Corollary discharge inhibition and preservation of temporal information in a sensory nucleus of mormyrid electric fish. J Neurosci 9:1029-1044.

Bell CC, Myers JP, Russell CJ (1974) Electric organ discharge patterns during dominance related behavioral displays in Gnathonemus petersii (Mormyridae). J Comp Physiol 92:201-228.

Bennett MV, Pappas GD, Aljure E, Nakajima Y (1967) Physiology and ultrastructure of electrotonic junctions. II. Spinal and medullary electromotor nuclei in mormyrid fish. J Neurophysiol 30:180-208.

Bennett MVL (1971) Electroreception. In: Fish physiology (Hoar WS, Randall DJ, eds), pp 493-574. London: Academic.

Buonomano DV (2000) Decoding temporal information: a model based on short-term synaptic plasticity. J Neurosci 20:1129-1141.

Cariani PA (2001) Neural timing nets. Neural Netw 14:737-753.

Carlson BA (2002) Electric signaling behavior and the mechanisms of electric organ discharge production in mormyrid fish. J Physiol Paris 96:405-419.

Carlson BA (2008) Temporal coding in electroreception. In: Encyclopedia of neuroscience (Binder MD, Hirokawa N, Windhorst U, Hirsch MC, eds), pp 4039-4044. New York: Springer.

Carlson BA, Hopkins CD (2004) Stereotyped temporal patterns in electrical communication. Anim Behav 68:867-878.

Carlson BA, Hopkins CD, Thomas P (2000) Androgen correlates of socially induced changes in the electric organ discharge waveform of a mormyrid fish. Horm Behav 38:177-186.

Carr CE (1986) Time coding in electric fish and barn owls. Brain Behav Evol 28:122-133.

Carr CE (1993) Processing of temporal information in the brain. Annu Rev Neurosci 16:223-243. 
Carr CE, Soares D (2002) Evolutionary convergence and shared computational principles in the auditory system. Brain Behav Evol 59:294-311.

Edwards CJ, Leary CJ, Rose GJ (2007) Counting on inhibition and ratedependent excitation in the auditory system. J Neurosci 27:13384-13392.

Edwards CJ, Leary CJ, Rose GJ (2008) Mechanisms of long-interval selectivity in midbrain auditory neurons: roles of excitation, inhibition, and plasticity. J Neurophysiol 100:3407-3416.

Eggermont JJ (1998) Is there a neural code? Neurosci Biobehav Rev 22:355-370.

Fortune ES, Rose GJ (2001) Short-term synaptic plasticity as a temporal filter. Trends Neurosci 24:381-385.

Grothe B (2003) New roles for synaptic inhibition in sound localization. Nat Rev Neurosci 4:1-11.

Grothe B, Klump GM (2000) Temporal processing in sensory systems. Curr Opin Neurobiol 10:467-473.

Hopkins CD (1986) Behavior of Mormyridae. In: Electroreception (Bullock TH, Heiligenberg W, eds), pp 527-576. New York: Wiley.

Hopkins CD, Bass AH (1981) Temporal coding of species recognition signals in an electric fish. Science 212:85-87.

Jagadeesh B, Wheat HS, Ferster D (1993) Linearity of summation of synaptic potentials underlying direction selectivity in simple cells of the cat visual cortex. Science 262:1901-1904.

Jeffress LA (1948) A place theory of sound localization. J Comp Physiol Psychol 41:35-39.

Kramer B (1974) Electric organ discharge interaction during interspecific agonistic behavior in freely swimming mormyrid fish a method to evaluate 2 or more simultaneous time series of events with a digital analyzer. J Comp Physiol A Neuroethol Sens Neural Behav Physiol 93:203-235.

Kramer B (1976) Electric signaling during aggressive behavior in Mormyrus rume (Mormyridae Teleostei). Naturwissenschaften 63:48-49.

Kramer B, Bauer R (1976) Agonistic behavior and electric signaling in a mormyrid fish, Gnathonemus petersii. Behav Ecol Sociobiol 1:45-61.
Large EW, Crawford JD (2002) Auditory temporal computation: interval selectivity based on post-inhibitory rebound. J Comput Neurosci 13:125-142.

Lestienne R (2001) Spike timing, synchronization and information processing on the sensory side of the nervous system. Prog Neurobiol 65:545-591.

Moller P (1976) Electric signals and schooling behavior in a weakly electric fish, Marcusenius cyprinoides L. (Mormyriformes). Science 193:697-699.

Moller P, Serrier J, Squire A (1979) Spacing and aggregation in mormyrid fish. Neurosci Lett 3:S60.

Rieke F, Warland D, de Ruyter van Steveninck R, Bialek W (1997) Spikes: exploring the neural code. Cambridge, MA: MIT.

Rose GJ, Fortune ES (1996) New techniques for making whole-cell recordings from CNS neurons in vivo. Neurosci Res 26:89-94.

Szabo T, Enger PS, Libouban S (1979) Electrosensory systems in the mormyrid fish, Gnathonemus petersii: special emphasis on the fast conducting pathway. J Physiol Paris 75:409-420.

Theunissen F, Miller JP (1995) Temporal encoding in nervous systems: a rigorous definition. J Comput Neurosci 2:149-162.

von der Emde G (1992) Electrolocation of capacitive objects in four species of pulse-type weakly electric fish: II. Electric signalling behaviour. Ethology 92:177-192.

Wong RY, Hopkins CD (2007) Electrical and behavioral courtship displays in the mormyrid fish Brienomyrus brachyistius. J Exp Biol 210:2244-2252.

Xu-Friedman MA, Hopkins CD (1999) Central mechanisms of temporal analysis in the knollenorgan pathway of mormyrid electric fish. J Exp Biol 202:1311-1318.

Zakon HH (1986) The electroreceptive periphery. In: Electroreception (Bullock TH, Heiligenberg W, eds), pp 103-156. New York: Wiley.

Zucker RS, Regehr WG (2002) Short-term synaptic plasticity. Annu Rev Physiol 64:355-405. 